\title{
High Dynamic Range Image Analysis through Various Tone Mapping Techniques
}

\author{
Manoj Kumar Patle \\ Scope college of Engineering, \\ Bhopal, India
}

\author{
Bharti Chourasia \\ Scope college of Engineering, \\ Bhopal, India
}

\author{
Yashwant Kurmi \\ Maulana Azad National \\ Institute of Technology, Bhopal \\ India
}

\begin{abstract}
The image quality is improved drastically with the increase of the technology. The conventional display devices may not be suitable for these High dynamic range images. The tone mapping is the process to show the good quality image in the normal LDR display devices. This paper presents a review of the tone mapping algorithms. It provides the methodology on Tone Mapped Image Quality Index (TMIQI) and the Blind Quality Assessment of Tone-Mapped Images (BTMQI). The region is basically expanded and compressed to visualize properly. Thereby the region-enhanced pseudo-exposures are fused into an HDR image. The image quality of BTMQI is comparatively higher than the TMIQI method. The low dynamic range images are suitable to both the conventional and advance display devices.
\end{abstract}

\section{Keywords}

High dynamic range imaging, structural preservation, tone mapping, perceptual image processing, structural similarity

\section{INTRODUCTION}

The today's era is a very flexible and may changes the requirements from place to place or city to city. The tone mapping is the process of making High dynamic range (HDR) images from the low dynamic range (LDR) images. The Inverse Tone Mapping us the process is the in which the HDR images are concerted into the LDR for images corresponding to the suitable display. There are various works has been done in this field. The one of the work done by Jinno et al. [1] using multiple exposure fusion to increase the dynamic range of an image. This method estimates displacements and occlusion and saturation regions simultaneously by using maximum a posteriori estimation and constructs motion-blur-free HDRIs. The exposer time selection consideration is the major parameter in this procedure. Bo Gu et al. [2] worked on decomposition at multiscale with this filter to manipulate a high dynamic range image, which has multiple detail layers and a base layer. The reproduced image gives a good visualization and preserving or enhancing local details.

Yeganeh et al. [3] worked on tone-mapping operators (TMOs) that make low dynamic range from high dynamic range images to visualize the HDR images on ordinary LDR displays. Different TMOs produce different tone-mapped images [4], with good quality. It presented an objective quality valuation system for tonemapped images by using multiscale signal fidelity through the modified structural similarity index and statistical parameters. Xiao et al. [5] proposed a graded tone mapping system based on colour appearance model. The Gaussian kernel is used to speed up the bilateral filter. The process of tone compression in RGB colour galaxy is accepted to correct the colour casts. The feature similarity index for tone-mapped images (FSITM) method, based on local phase indication of images is evolved for tone mapping. Its index matches the locally weighted mean phase angle map [6] of an original high dynamic range (HDR) to its associated tone-mapped image calculated using the output of the TMO method. Wang et al. [7] worked on a region-based boost of the pseudo-exposures to produce an HDR image. It considers a region-based enrichment on pseudo-exposures to boost details in the most distinct region. The fusion is then applied for the region-enhanced pseudoexposures to create an HDR image.

Ma et al. [8] worked on tone mapping operators (TMOs) aim to directly circumnavigates the space of all images, searching for the image that optimizes an improved TMQI. Specifically, it improves structural fidelity and statistical naturalness. It is an iterative algorithm that alternatively improves the statistical naturalness and structural fidelity of the resulting image. K. Gu et al. [9] developed an effective and efficient reference less objective quality metric which can assess LDR images created by different TMOs without access to the original HDR images. The basic approach are considered for tone mapping from texture based tone mapping [10-15] and tone mapping for backlight scaling [16].

This paper reviews the work on tone mapping for the HDR image to display in conventional devices. The second section provides the methodology on iterative tone mapping and the Blind Quality Assessment of Tone-Mapped Images (BTMQI). The third section shows the result analysis and fourth section covering future scope with in conclusion.

\section{METHODOLOGY}

\subsection{Iterative Tone Mapping by Optimizing TMQI-II [8]}

Let the $\mathrm{X}$ is the HDR image and $\mathrm{Y}$ is the tone mapped LDR image. Then the tone mapping method TMQI suggests a tone mapped image of good quality to achieve excessive structural fidelity corresponding to the HDR image. The TMQI calculation is specified by [3]

$$
\operatorname{TMQI}(X, Y)=a[S(X, Y)]^{\alpha}+(1-a)[N(Y)]^{\beta}
$$

Where $\mathrm{S}$ represents the structural fidelity and $\mathrm{N}$ represents the naturalness measure of the image. The sensitivities of the two terms are determined by the parameters $\alpha$ and $\beta$. The relative importance between $\alpha$ and $\beta$ is regulated by the value of a: $\{0$ $\leq \mathrm{a} \leq 1\}$. Here $S \leq 1$ and $N \leq 1$ hence the value of TMQI is bounded as $T M Q I \leq 1$. The attempt for image quality estimation across dynamic range is achieved by TMQI. Here, TMQI-II as the upgraded version of TMQI is discussed that overcomes the and better correlate through subjective calculations. TMQI-II is elaborated with the statistical naturalness components and structural fidelity.

For the understanding of the algorithm assume that TMQI-II creates quality in tone mapped images, the formulation of optimal tone mapping can be expressed as 


$$
Y_{o p t}=\underset{Y}{\arg \max } T M Q I-I I(X, Y)
$$

Here the range $\mathrm{Y}$ is less dynamic than $\mathrm{X}$. the solution of (2) to get $Y_{\text {opt }}$ is a challenging task because of the complication of TMQI-II method and the high dimensionality. It is implemented with the optimization approach to improve the result through iterative approach. The initial image $\mathrm{Y0}$ is considered as input. It is moved in the spatial domain in the direction that improves quality of TMQI-II. The overall approach is divided in two parts for ease of understanding. It uses the gradient ascent method to upgrades the structural fidelity $\mathrm{S}$ and then by intensity parameter optimization boosts the statistical naturalness $\mathrm{N}$. The procedure constitutes iteration and the iterations continue until convergence.

\subsubsection{Structural Fidelity Update}

The TMQI structural fidelity is calculated with the sliding frame across the complete image, as a outcome a quality map is generated that consists the local structural conservancy. The structural fidelity measure is computed using the covariance between the two corresponding patches and local standard deviations with some positive constants to avoid instability. The overall fidelity is the average of all the local structural fidelity measure.

\subsubsection{Statistical Naturalness Update}

The naturalness $\mathrm{N}$ in terms of statistical parameter in TMQI is created by modeling $\mu$ and $\sigma$ histograms of approx. 3000 natural scenes by a density function of Gaussian nature $P_{m}$ and a Beta function $P_{d}$, respectively [3]. The global statistical naturalness measure is defined as

$$
\mathrm{N}(\mathrm{Y})=\frac{1}{\mathrm{~K}} \mathrm{P}_{\mathrm{m}} \cdot \mathrm{P}_{\mathrm{d}}
$$

The logarithmic function is also applied for the range adjustment by assuming that most structural detail in HDR image present in a low dynamic range and thus it is reasonable to boost lower luminance range with compressing the higher luminance range.

\subsection{BLIND BTMQI Approach [9]}

There is the tone mapping algorithm that searches the informational parts in the image. The blind quality assessment method for tone-mapping provides good visual difference between the images of low and high dynamic range.

It is found that the images generated by tone-mapping, have constraint of dynamic range and cannot preserve the original HDR image information. On this basis of metric calculation the tone-mapping method straightforwardly estimate the image quality information in itself. The transitional images created by the low intense/ high intense change in the original luminance. The transitional images are created by

$$
I_{i}=\min \left(\max \left(M_{i} . I, 0\right), 255\right)
$$

where I represents tone-mapped image as input, and Mi specifies ith multiplier. The operators for minimum and maximum calculation as max and min are applied to clip the transitional images into range of $0 \sim 255$. The further step is to measure the information amount using various parameters. The entropy of data [17] is concept in statistics, for appropriate parameter selection criterion. The probability calculation of any one signal of random nature the entropy is the measure of disorder degree of it. The given probability density $p$ in exact manner define its entropy as

$$
E(p)=-p(x) \log p(x) d x
$$

It is found by experiments that, the entropy uses the K-L provide the considerable gain performance and then introduce much time complexity. So it is determined to adopt the entropy for quantifying the information. In most cases a good tone-mapped image, is generally of larger entropy.

To achieve good result efficiency the advantage of only nine entropy values that are considered here and measured using divergence or its symmetric versions. Those are not able to

$$
M=\left\{1, n, \frac{1}{n} \mid n=3.5,5,6.5,8\right\}
$$

It is can be tested for numerous other values of $\mathrm{n}$ and result shows that about $2 \%$ performance improvements relative to the most of the choices. The entropy of each of nine intermediate images is redefined as

$$
\mathrm{E}_{\mathrm{t}}\left(I_{i}\right)=w E_{g}\left(I_{i}\right)+(1-w) E_{l}\left(I_{i}\right)^{v}
$$

where $E_{g}\left(I_{i}\right)$ is the global entropy and $E_{l}\left(I_{i}\right)$ represents the local entropy; $\mathrm{w}$ and $\mathrm{v}$ are positive constants to control the relative significance of the two constituents are set to be 0.59 and 1.5 as an empirical value. The local entropy is calculated through the mean value of block-based entropy [18], which is defined by

$$
E_{l}\left(I_{i}\right)=\frac{1}{L} \sum_{j=1}^{L} E\left(B_{i, j}\right)
$$

where $\boldsymbol{B}_{i, j}$ indicates the jth block of size $72 \times 72$ in the ith intermediary image; and value of $L$ represents the number of blocks in image. Another consideration of this method is the statistical naturalness; the outcome is considered as the good quality tone mapped natural LDR image.

There exist lots of NR-IQA metrics from the literature that were built on the natural scene statistics, e.g., [19]-[21]. The basic thought behind these simulations present in that, shown for natural images, the coefficients processed in normalized form by local mean removal with normalized divisive as Gaussian distribution, however various distortion types or levels will restructure this distribution.

\section{EXPERIMENTAL RESULTS AND ANALYSIS}

The result analysis is shown through the visual illustration in figure 1 and the parameter comparison as illustrated in table 1 . The parameters considered are discussed in this section as the Spearman's rank-order correlation coefficient (SRCC) and Kendall's rankorder correlation coefficient (KRCC) for the evolution of the above discussed algorithms [3]. The parameters are formulated as

$$
S R C C=1-\frac{6 \sum_{i=1}^{N} d_{i}^{2}}{N\left(N^{2}-1\right)}
$$

where ${ }^{d_{i}}$ represents the difference among the ranks of i-th image in objective and subjective evaluations. The statistical parameters are very suitable for the comparison of different method. 


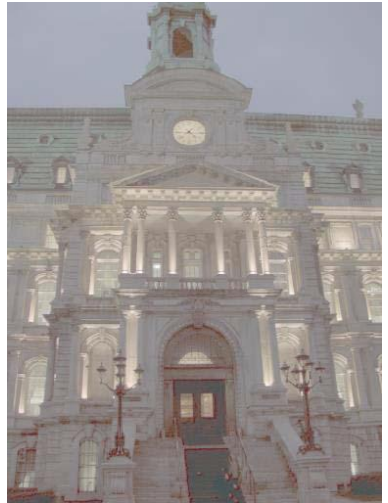

(a)

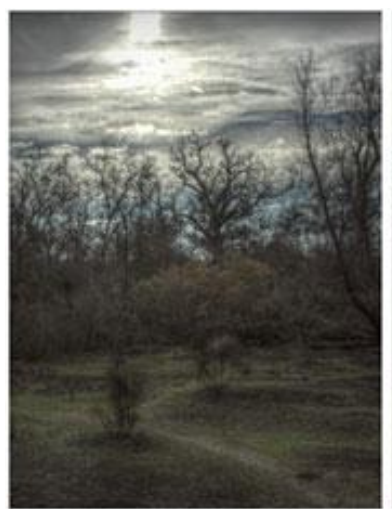

(d)

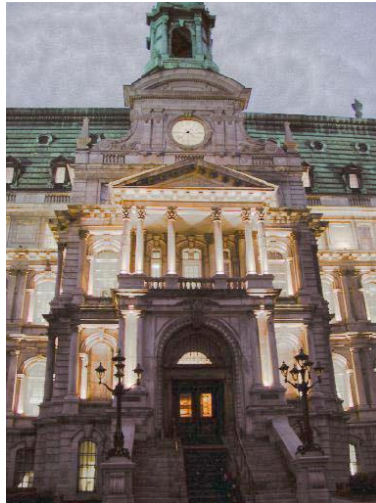

(b)

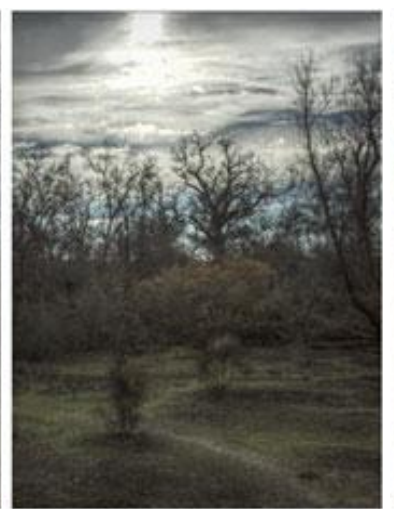

(e)

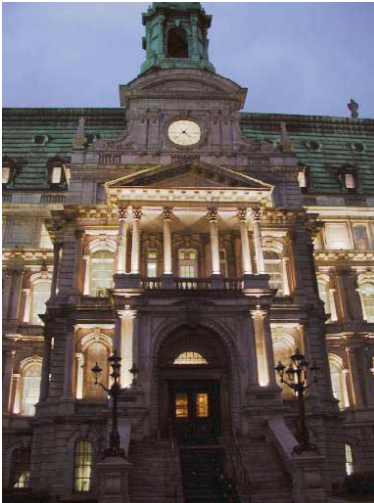

(c)

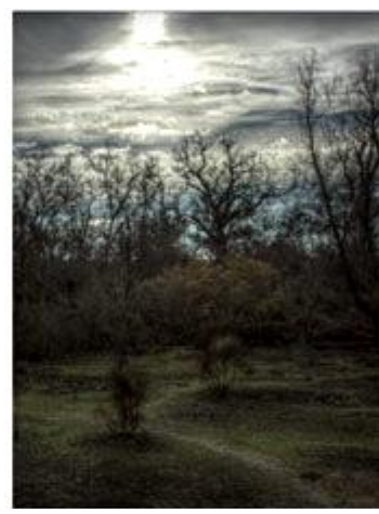

(f)

Fig 1: The images of Clock building and forest (a) and (d) shows the original images, (b) and (e) represents the Iterative Tone Mapping results and (c) and (f) shows the result by blind BTMQI.

Table 1. The SRCC and the KRCC parameter comparison for the two Clock building and forest images

\begin{tabular}{|c|c|c|c|}
\hline \multirow{2}{*}{ Images } & Method & SRCC & KRCC \\
\hline $\begin{array}{c}\text { Clock Building } \\
\text { Image }\end{array}$ & TMIQI & 0.7471 & 0.6030 \\
\cline { 2 - 4 } & BTMQI & 0.7882 & 0.6219 \\
\hline Forest Image & TMIQI & 0.7482 & 0.6132 \\
\cline { 2 - 4 } & BTMQI & 0.8262 & 0.6611 \\
\hline
\end{tabular}

$$
K R C C=\frac{N_{c}-N_{d}}{\frac{1}{2} N(N-1)}
$$

where $N_{c}$ is the numbers of concordant of consistent rank order and $N_{d}$ is the numbers of discordant of inconsistent rank order pairs with each other in the data set. The table 1 shows the quantitative comparison of both the algorithms. The BTMQI method gives the superior result as compared to the TMIQI method. The BTMQI method gives the $8 \%$ more SRCC parameter as compared to TMIQI for the clock building image. The BTMQI method gives the $8 \%$ more SRCC parameter as compared to TMIQI for the forest image.

\section{CONCLUSION}

This paper presents a review of the tone mapping algorithms. It provides the methodology on Tone Mapped Image Quality Index (TMIQI) and the Blind Quality Assessment of ToneMapped Images (BTMQI). The region is basically expanded and compressed to visualize properly. Thereby the regionenhanced pseudo-exposures are fused into an HDR image.
The image quality of BTMQI is comparatively higher than the TMIQI method. The low dynamic range images are suitable to both the conventional and advance display devices. The overall performance of BTMQI method is $9 \%$ superior in terms of SRCC parameter as compared to TMIQI.

The fast search algorithms are the most important and necessary requirements of the future work. The next objective is quality assessment of tone mapped images in terms of various parameters.

\section{REFERENCES}

[1] T. Jinno, and M. Okuda, "Multiple exposure fusion for high dynamic range image acquisition," IEEE Trans. on Image Process., vol. 21, no. 1, Jan. 2012, pp. 358-365.

[2] B. Gu, W. Li, M. Zhu, and M. Wang, "Local edgepreserving multiscale decomposition for high dynamic range image tone mapping," IEEE Trans. on Image Process., vol. 22, no. 1, Jan. 2013, pp. 70-79.

[3] H. Yeganeh and Z. Wang, "Objective quality assessment of tone-mapped images," IEEE Trans. on Image Process., vol. 22, no. 2, Feb. 2013, pp. 657-667.

[4] A. Chakrabarti, Y. Xiong, B. Sun, T. Darrell, D. Scharstein, T. Zickler, and K. Saenko, "Modeling radiometric uncertainty for vision with tone-mapped color images," IEEE Trans. on Pattern Analysis And Machine Intelligence, vol. 36, no. 11, Nov. 2014, pp. 2185-2198.

[5] J. Xiao, W. Li, G. Liu, S. L. Shaw, Y. Zhang, "Hierarchical tone mapping based on image colour 
appearance model," IET Comput. Vis., 2014, vol. 8, no. 4, pp. 358-364.

[6] H. Z. Nafchi, A. Shahkolaei, R. F. Moghaddam, and M. Cheriet, "FSITM: A feature similarity index for tonemapped images," IEEE Signal Process. Letters, vol. 22, no. 8, Aug. 2015, pp.1026-1029.

[7] T. H. Wang, C. W. Chiu, W. C. Wu, J. W. Wang, C. Lin, C. T. Chiu, and J.J. Liou, "Pseudo-multiple-exposurebased tone fusion with local region adjustment," IEEE Trans. on Multimedia, vol. 17, no. 4, Apr. 2015, pp.470484.

[8] K. Ma, H. Yeganeh, K. Zeng, and Z. Wang, "High dynamic range image compression by optimizing tone mapped image quality index," IEEE Trans. on Image Process., vol. 24, no. 10, Oct. 2015, pp. 3086-3097.

[9] K. Gu, S. Wang, G. Zhai, S. Ma, X. Yang, W. Lin, W. Zhang, and Wen Gao, "Blind quality assessment of tonemapped images via analysis of information, naturalness, and structure," IEEE Trans. on Multi., vol. 18, no. 3, Mar. 2016, pp. 432-443.

[10] T. Tan and A. G. Constantinides, "Multi-Slice Image Texture Edge Detection by Local Vector Mapping," IEEE, 1988, pp. 1136-1139.

[11] Y. Kurmi and V. Chaurasia, "An image fusion approach based on adaptive fuzzy logic model with local level processing," Int. Jour. of Comp. Appl., Aug. 2015, vol. 124, no.1, pp. 39-42.

[12] D. Sharma, Y. Kurmi, and V. Chaurasia, "Formation of super- resolution image: a review," Int. Jour. of Emerging Tech. and Adv. Engg., Apr. 2014, vol. 4, no. 4, pp. 218221.
[13] Y. Kurmi and V. Chaurasia, "Performance of haze removal filter for hazy and noisy images," Int. Jour. of Sci. Engg. and Tech., Apr. 2014, vol. 3 no. 4, pp. 437439.

[14] S. Tiwari, K. Chauhan, and Y. Kurmi "Shadow detection and compensation in aerial images using MATLAB," Int. Jour. of Comp. Appl., June 2015, vol. 119, no.20, pp. 5-9.

[15] T. S. Huang, "Coding of two tone images," IEEE Trans. on Commu., Nov. 1977, vol. Com-25, no. 11, pp. 14061424.

[16] A. lranli and M. Pedram, "DTM: Dynamic Tone Mapping for Backlight Scaling," Anaheim, California, USA, DAC June 13-17, 2005, pp. 612-617.

[17] C. E. Shannon, "A mathematical theory of communication," Bell Syst. Tech. J., vol. 27, no. 3, pp. 379-423, Oct. 1948.

[18] W. Xue, L. Zhang, and X.Mou, "Learning without human scores for blind image quality assessment," in Proc. IEEE Int. Conf. Comput. Vis. Pattern Recog., Jun. 2013, pp. 995-1002.

[19] A. K. Moorthy and A. C. Bovik, "Blind image quality assessment: From scene statistics to perceptual quality," IEEE Trans. Image Process., vol. 20, no. 12, pp. 33503364, Dec. 2011.

[20] M. A. Saad, A. C. Bovik, and C. Charrier, "Blind image quality assessment: A natural scene statistics approach in the DCT domain," IEEE Trans. Image Process., vol. 21, no. 8, pp. 3339-3352, Aug. 2012.

[21] A. Mittal, A. K. Moorthy, and A. C. Bovik, "Noreference image quality assessment in the spatial domain," IEEE Trans. Image Process., vol. 21, no. 12, pp. 4695-4708, Dec. 2012. 